\title{
3D Collision Avoidance Algorithm for Unmanned Aerial Vehicles with Limited Field of View Constraints
}

\author{
Steven Roelofsen ${ }^{1,2}$, Alcherio Martinoli ${ }^{1}$ and Denis Gillet ${ }^{2}$
}

\begin{abstract}
Unmanned Aerial Vehicles (UAVs) are becoming a significant field of research with numerous applications, ranging from mapping to surveillance. New applications, such as aerial delivery of goods, are expected to appear in the next years and will require more and more autonomy from UAVs. One challenge preventing UAVs from being fully autonomous is their current limitations in handling potential collisions among multiple vehicles. This paper presents a collision avoidance algorithm for fixed-wing UAVs navigating in a three dimensional space. It satisfies limited field of view constraints that stem from the use of a single camera system as sensing device. The proposed algorithm uses potential fields to both navigate and avoid obstacles. To guarantee collision avoidance, the algorithm is enhanced with a turning behavior that allows for ensuring the safety of the method. Simulations are performed to show the effectiveness of the proposed algorithm.
\end{abstract}

\section{INTRODUCTION}

Recently, the field of small Unmanned Aerial Vehicles (UAVs) became of significant importance as numerous new applications, ranging from mapping to surveillance, appeared. For example, aerial delivery of goods is expected to appear in near future and will require increasingly more autonomy. Up to date, research activities focused mainly on selflocalization [1], path planning [2], [3] and navigation [4], [5]. Importantly, ensuring safety is one of the remaining challenges that needs to be overcome in order to achieve fully autonomous UAVs. More specifically, the UAVs do not have yet the capacity to avoid collisions with other threats, including other UAVs. The challenge is even greater if UAVs do not mutually share their positions, and have to rely only on on-board sensors to detect and track threats.

Currently, research considers several sensing modalities that allow for tracking other UAVs, including vision using on-board cameras. Visual sensing is of particular interest as it generally requires less power and is lighter than other solutions. It also enables to track other aircraft without the latter requiring specific equipment (e.g., transponders). One downside of the use of cameras is their limited Field Of View (FOV). Although a camera can have a FOV larger than $180^{\circ}$ using a fish-eye lens, it will always have a blind spot. It is possible to add several cameras pointing in different directions to cover the whole space or use pan-and-tilt cameras, but

This work has been financially supported by Honeywell, and has benefitted of the administrative and technical coordination of the EPFL Transportation Center.

1 S. Roelofsen and A. Martinoli are with the Distributed Intelligent Systems and Algorithms Laboratory, School of Architecture, Civil and Environmental Engineering, École Polytechnique Fédérale de Lausanne.

${ }^{2}$ S. Roelofsen and D. Gillet are with the the Coordination and Interaction System Group, School of Engineering, École Polytechnique Fédérale de Lausanne. it would be at the expense of increased weight. As a result, the collision avoidance algorithm not only needs to satisfy actuator constraints, but also take into account the limitations of the chosen sensor modality.

Several contributions on collision avoidance systems for UAVs have been published over the last few years. In [6] navigation functions are used to avoid collision between autonomous vehicles under actuation and sensing constraints, although not limited in FOV. Avoidance maneuvers for aircraft have been presented in [7] and validated for constrained sensing (both in range and in FOV), although no analytical proof of collision avoidance is presented. In [8] the authors introduce a gyroscopic force field to improve the collision avoidance capability of the navigation function for holonomic agents. In [9] a similar approach is used and applied to aircraft with limited turning rate and sensing range. The two latter studies rely on introducing a gyroscopic force to the potential field gradient to avoid stagnation points and guarantee collision avoidance.

A number of algorithms has been validated experimentally. In [10] an algorithm based on velocity obstacles has been implemented on quadrotors, and while the algorithm is competitive in minimising the change in motion to perform an avoidance, neither the algorithm nor the camera FOV can guarantee avoidance in all situations. The algorithm presented in [11] leverages the properties of spherical lenses by keeping the object to be avoided on one edge of the image. Even if the authors show a proof of collision avoidance, they assume the object is already in the FOV. Moreover, they do not show a proof of reciprocal collision avoidance. In other words, none of these works explicitly addresses the FOV problem. Our previous work [12] is, to our knowledge, the first collision algorithm that takes into account the FOV limitations and has been experimentally validated [13].

In this paper, we will present a three-dimensional collisionavoidance algorithm that satisfies the limited FOV constraints of cameras for fixed-wing aircraft. We introduce the requirements to guarantee collision avoidance using the proposed algorithm and show a proof of its safety. We also compare the algorithm's performance with previous work published in [12] in terms of turn rate. This metric is of importance as turning rate is the only command input that is not bounded in our algorithm.

The paper is structured as follows: Section II describes the system of interest. Section III presents the potential field used for navigation. Section IV and V present the control laws for the system, and show proofs. Simulations of the algorithm are presented in Section VI. 


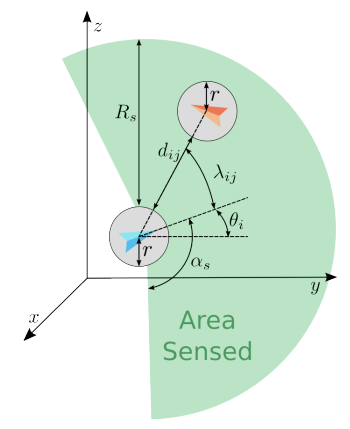

Fig. 1. Illustration of the angles and distances used in this paper. The green area represents the area where aircraft $j$ (in orange) is sensed by aircraft $i$ (in blue). Note that the angles $\theta_{i}$ and $\lambda_{i j}$ might not be in the same plane. The FOV is defined by $\alpha_{s}$ in all direction (thus both horizontally and vertically).

\section{Problem Formulation}

In this paper we consider $N$ non-holonomic homogeneous vehicles in a three dimensional space $[x, y, z]$ with dynamics similar to fixed-wing aircraft. Their motion is a combination of an unicycle model on the horizontal plane and velocity control on the vertical axis:

$$
\left[\begin{array}{c}
\dot{x}_{i} \\
\dot{y}_{i} \\
\dot{z}_{i} \\
\dot{\theta}_{i}
\end{array}\right]=\left[\begin{array}{c}
v_{i} \cos \theta_{i} \\
v_{i} \sin \theta_{i} \\
l_{i} \\
u_{i}
\end{array}\right]
$$

with $\mathbf{q}_{i}=\left[x_{i}, y_{i}, z_{i}\right]$ vehicle's position and $\theta_{i}$ its heading (defined on the horizontal plane). The heading and any other angle considered in this paper are always defined between $[-\pi ; \pi]$ and any function returns angles in that interval. The aircraft can be controlled with the three input variables $u_{i}$, $l_{i}$, and $v_{i}$ that are the yaw turning rate, the vertical speed, and the forward speed, respectively. The horizontal speed $v_{i}$ is constrained by:

$$
0<v_{-} \leq v_{i} \leq v_{+}
$$

with $v_{+}$the maximum horizontal speed and $v_{-}=v_{+}-\Delta v$ the minimum horizontal speed of the aircraft.

An aircraft $i$ goes from a starting point $q_{o_{i}}$ to a destination point $q_{d_{i}}$, while avoiding collisions with other aircraft. The collision zone of each aircraft $i$ is a sphere of radius $r$ that fully includes the aircraft. The collision zone is a zone that should not be crossed by any obstacle or other aircraft. A collision is defined between aircraft $i$ and $j$ if the distance between the two aircraft is smaller than $2 r$.

Aircraft $i$ is capable to sense an aircraft $j$ that is located at a distance $d_{i j}=\left\|\mathbf{q}_{i}-\mathbf{q}_{j}\right\|-2 r<R_{s}$ and at an angle $\lambda_{i j} \leq \alpha_{s}$ with $\alpha_{s}>\pi / 2$ where

$$
\lambda_{i j}=\arccos \left(\frac{\left[\cos \theta_{i}, \sin \theta_{i}, 0\right]^{T} \cdot\left(\mathbf{q}_{i}-\mathbf{q}_{j}\right)}{\left\|\mathbf{q}_{i}-\mathbf{q}_{j}\right\|}\right) .
$$

The definitions are illustrated in Figure 1.

\section{NAVIGATION}

The proposed control scheme is based on potential field theory but adapted to the FOV constraints of our system. The proposed potential field is a combination of a function $V_{i}^{\text {nav }}$ that steers aircraft $i$ to its destination, and another function $V_{i j}^{a v o i d}$ that steers aircraft $i$ away from another aircraft $j$. The function that steers aircraft $i$ to destination is defined as:

$$
V_{i}^{n a v}=K_{n a v}\left\|\mathbf{q}_{i}-\mathbf{q}_{d_{i}}\right\|^{2}
$$

but other functions are possible [9]. The collision avoidance function is defined as:

$$
V_{i j}^{\text {avoid }}=\frac{K_{\text {avoid }}}{\left\|\mathbf{q}_{i}-\mathbf{q}_{j}\right\|-2 r} .
$$

Both $K_{\text {avoid }}$ and $K_{\text {nav }}$ are gains. Like in our previous work [12], the aircraft $i$ is directed by the weighted sum of the gradient of the potential fields described in Equations 4 and 5 in the following manner:

$$
\begin{aligned}
\nabla V_{i}^{\text {tot }} & =\left(1-\max _{j}\left(\beta_{a_{j}} \beta_{d_{j}}\right)\right) \nabla V_{i}^{\text {nav }} \\
& +\sum_{j} \beta_{a_{j}} \beta_{d_{j}} \nabla V_{i j}^{\text {avoid }} .
\end{aligned}
$$

From Equation 6 we also define the desired direction for aircraft $i$ as $-V_{i}^{t o t}=\left[x_{t, i}, y_{t, i}, z_{t, i}\right]^{T}$. The weighting function $\beta_{a_{j}}$ and $\beta_{d_{j}}$ encode aircraft's sensors limitations, and are defined as:

$$
\begin{aligned}
& \beta_{d_{j}}=\beta\left(\frac{R_{s}-d_{i j}}{R_{s}-R_{a}}\right), \\
& \beta_{a_{j}}=\beta\left(\frac{\alpha_{s}-\left|\lambda_{i j}\right|}{\alpha_{s}-\frac{\pi}{2}}\right)
\end{aligned}
$$

with $R_{a}$ the distance at which an aircraft should only be avoiding. The barrier function $\beta$ is defined as follows:

$$
\beta(a)= \begin{cases}0 & \text { if } a<0 \\ f(a) & \text { if } 0 \leq a<1 \\ 1 & \text { otherwise }\end{cases}
$$

with $f(a)$ a monotonic and smooth function that allows a smooth transition of $\beta$ from 0 to 1 . In this paper $f(a)$ is similar to the one used in [14] and is defined as:

$$
f(a)=3 a^{2}-2 a^{3}
$$

The potential field cannot be directly applied to the three inputs that are $u_{i}, l_{i}$, and $v_{i}$ for two reasons. First, the relation between those commands and the aircraft's state space is nonlinear and thus the gradient of the potential field needs to be translated into those inputs. Second, the potential field alone does not provide collision avoidance guarantees because of the non-holonomicity of the system. As a consequence, the three inputs are controlled separately as described in the following section.

\section{CONTROL}

This section describes how to convert the potential field to command input for the aircraft. The turning rate command $u_{i}$ performs the horizontal avoidance, and is the most important component in collision avoidance. The vertical speed command $l_{i}$ is responsible for height control and vertical collision avoidance, although the latter is limited by the FOV. Finally, the horizontal speed command $v_{i}$ is responsible for faster avoidance resolution as reported in [12]. 


\section{A. Speed control}

As explained in [12], a change in forward speed $v_{i}$ is required to mitigate deadlocks. Even if having two aircraft at exactly the same speed and height is unlikely, we reuse the same control law to improve system's separation capability.

$$
v_{i}=v_{+}-\max _{j}\left(\beta_{a_{j}} \beta_{d_{j}}\right) \Delta v
$$

\section{B. Height control}

As the height dynamics is decoupled from the other state variables, it would be possible to directly apply the negative potential field's gradient $-\nabla V_{i}^{\text {tot }}$ to the vertical speed $l_{i}$. But due to FOV constraints a saturation $l_{s a t}$ on the vertical speed is required (see Proposition 1).

$$
0<l_{\text {sat }}=v_{i} \sin \left(\alpha_{s}-\frac{\pi}{2}-\rho\right)<v_{i} \sin \left(\alpha_{s}-\frac{\pi}{2}\right)
$$

and the maximum vertical speed is :

$$
l_{\text {max }}=v_{+} \sin \left(\alpha_{s}-\frac{\pi}{2}-\rho\right)
$$

with $0<\rho<\alpha_{s}-\frac{\pi}{2}$ a margin angle. The absolute maximum speed is $v_{\max }=\sqrt{v_{+}^{2}+l_{\text {max }}^{2}}$. The saturation is applied using the following equation:

$$
l_{i}=\min \left(\max \left(K_{z} z_{t, i},-l_{\text {sat }}\right), l_{\text {sat }}\right)
$$

with $K_{z}$ a strictly positive gain.

\section{Yaw control}

The yaw control ${ }^{1}$ is responsible of steering the aircraft away from threat on the horizontal plane. It switches between following the potential field and a simple collision avoidance behavior.

$$
\begin{aligned}
u_{i}=\quad & K_{a}\left(1-\max _{j}\left(\beta_{a_{j}} \beta_{d_{j}}\right)\right)\left(\theta_{t}-\theta_{i}\right) \\
& +\sum_{j} \beta_{a_{j}} \beta_{d_{j}} \frac{-2 \pi v_{\max }}{d_{i j}} .
\end{aligned}
$$

with the target heading $\theta_{t}=\operatorname{atan} 2\left(y_{t, i}, x_{t, i}\right)$, and $K_{a}$ a gain.

\section{Collision Avoidance}

One of the challenges of collision avoidance under limited FOV constraints is to design a control law that does not generate a trajectory that would lead aircraft to a collision with none of them being able to sense a priori its related threat. This in turn enforces constraints on the shape of the FOV itself; indeed, this is the reason why the FOV in this paper is hemispherical with an angle strictly larger than $\pi$ radian. However, note that even with the FOV satisfying these constraints, poorly designed control law can lead to collisions. In this subsection, we show that the proposed control law does not generate trajectories that lead to collisions with the help of two propositions and corresponding proofs. The first proof demonstrates that if two aircraft do not sense each other, they are no able to collide with the proposed control

\footnotetext{
${ }^{1}$ The yaw control described by Equation 15 is slightly different from the work in [12]. This is to correct for a mistake in [12] where the turn rate is only half what would be needed to prove avoidance. The proof in [12] is also correct if the avoidance term in the yaw control is multiplied by two.
}

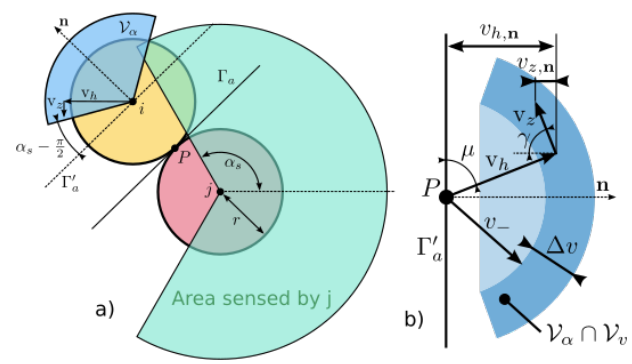

Fig. 2. Illustration of the variables involved in Proposition 1. a) Two aircraft about to collide, defining $\Gamma_{a}$ and $\mathcal{V}_{\alpha}$. b) Computation of the projections of $\mathbf{v}_{h}$ and $\mathbf{v}_{z}$ on $\mathbf{n}$.

laws. The second proof shows that if they mutually sense, then the control law maneuvers the aircraft safely away from the potential collision.

Proposition 1: An aircraft with FOV described by Equation 3, its dynamics described by Equation 1 and the control law described by Equations 11, 13 and 15 will not move along a trajectory that would lead to a collision with another aircraft without them being able to sense each other. That is, there is no possible collision without at least one of the two aircraft sensing the other aircraft.

Proof: Consider two UAVs $i$ and $j$ that are about to collide at point $P$ while none of the two is sensing the other one. Consider also the plane $\Gamma_{a}$ that goes through $P$ and is tangent to the two collision spheres of aircraft $i$ and $j$. To collide, at least one of the two aircraft needs to move towards the plane $\Gamma_{a}$. We will prove that with the proposed control law it is impossible for any of the UAVs to move towards $\Gamma_{a}$ without sensing the other UAV.

Without loss of generality, the proof can be done while fixing the frame of one of the aircraft, for example aircraft $j$ as shown in Figure 2a. As none of the two can see each other, the point $P$ is on the sphere sector of $j$ that is not in the FOV of aircraft $j$. Placing $P$ also defines the position of aircraft $i$ relative to aircraft $j$. Define $\mathcal{V}_{\alpha}$ as the cone formed by all camera directions for which aircraft $i$ does not see aircraft $j$. Define also $\Gamma_{a}^{\prime}$ the plane parallel to $\Gamma_{a}$ located at the center of $i$. Note that aircraft $i$ not crossing $\Gamma_{a}$ is equivalent to the center of $i$ moving away from aircraft $j$. Because it is solely defined by the geometry of the FOV of $j, \mathcal{V}_{\alpha}$ is always the same relative to $\Gamma_{a}^{\prime}$ : it is a right circular cone with its apex centered on $i$, its axis normal to $\Gamma_{a}^{\prime}$ and an aperture of $\pi-2\left(\alpha_{s}-\frac{\pi}{2}\right)$.

Because the horizontal velocity $\mathrm{v}_{h}=v_{i}\left[\cos \theta_{i}, \sin \theta_{i}, 0\right]^{T}$ is aligned with the camera, we have that $\mathrm{v}_{h} \in \mathcal{V}_{\alpha} \cap \mathcal{V}_{v}$ with $\mathcal{V}_{v}$ the spherical shell formed by the velocities between $\left[v_{-}, v_{+}\right]$. The shape of $\mathcal{V}_{\alpha} \cap \mathcal{V}_{v}$ is shown in Figure 2b. Because $\alpha_{s}>\pi / 2$ the velocity $\mathrm{v}_{h}$ alone will never lead to a collision when both aircraft do not see each other and can only be due to the vertical velocity $\mathrm{v}_{z}=\left[0,0, l_{i}\right]^{T}$. Define $\mathbf{n}$ the vector normal to $\Gamma_{a}^{\prime}$ and directed away from aircraft $j$, and project $\mathrm{v}_{h}$ and $\mathrm{v}_{z}$ to obtain $v_{h, \mathbf{n}}$ and $v_{z, \mathbf{n}}$ respectively. We want to show that:

$$
\left\|v_{h, \mathbf{n}}\right\|>\left\|v_{z, \mathbf{n}}\right\| \quad \forall v_{h, \mathbf{n}} \in \mathcal{V}_{\alpha} \cap \mathcal{V}_{v}, l_{i}
$$



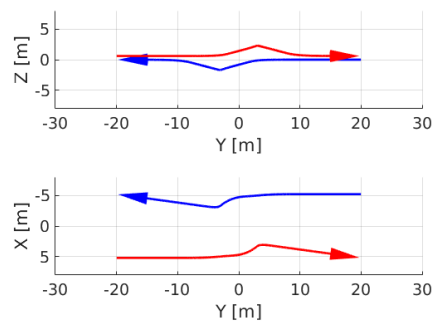

(a)
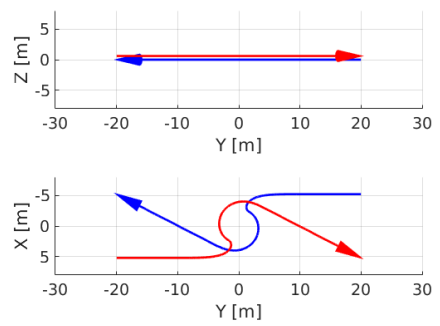

(d)
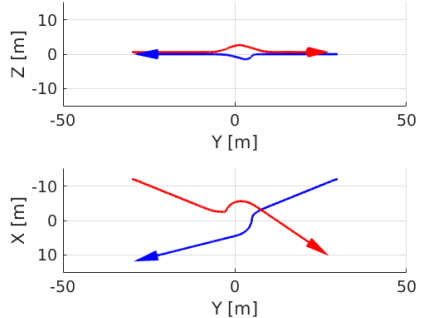

(b)
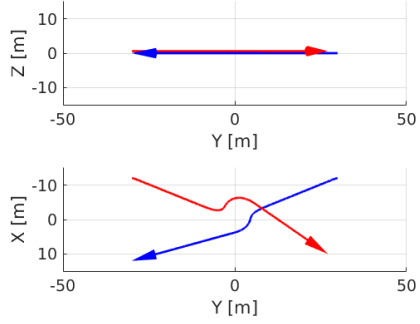

(e)
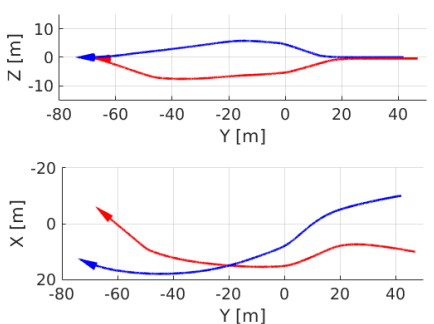

(c)
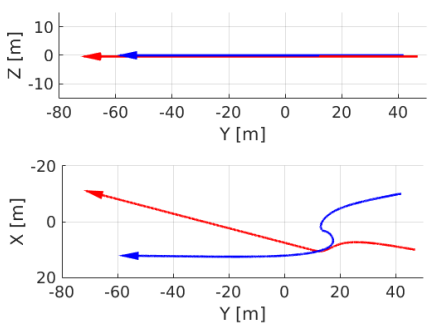

(f)

Fig. 3. Top row $(a, b, c)$ : three sets of trajectories obtained with presented collision avoidance algorithm. Bottom row (d,e,f): three sets of trajectories obtained with the algorithm from previous work [12] for the same initial conditions.

First, define the angle $\mu$ between the plane $\Gamma_{a}^{\prime}$ and the vector $\mathrm{v}_{h}$. Note that $\pi / 2>\mu \geq \alpha_{s}-\pi / 2$. The left hand of Equation 16 is:

$$
\left\|v_{h, \mathbf{n}}\right\|=\left\|\mathrm{v}_{h}\right\| \sin (\mu)=v_{i} \sin (\mu) \geq v_{i} \sin \left(\alpha_{s}-\pi / 2\right)
$$

Similarly, by defining the angle $\gamma$ between $v_{z, \mathbf{n}}$ and $-\mathbf{n}$, the right hand of Equation 16 becomes:

$$
\left\|v_{z, \mathbf{n}}\right\|=\left\|\mathrm{v}_{z}\right\| \cos (\gamma)<\left\|\mathrm{v}_{z}\right\| \leq l_{\text {sat }}=v_{i} \sin \left(\alpha_{s}-\frac{\pi}{2}-\rho\right)
$$

with $\pi / 2>\gamma \geq \alpha_{s}-\pi / 2$. Combining Equations 17 and 18 the final result is obtained:

$$
\left\|v_{h, \mathbf{n}}\right\|>v_{i} \sin \left(\alpha_{s}-\frac{\pi}{2}\right)>v_{i} \sin \left(\alpha_{s}-\frac{\pi}{2}-\rho\right)>\left\|v_{z, \mathbf{n}}\right\|
$$

Thus aircraft $i$ subject to control law described by Equations 11,13 and 15 will move away from aircraft $j$ if unable to sense aircraft $j$ and as a result will not be responsible for colliding. The same reasoning can be done for aircraft $j$ and as a result both aircraft are unable to move towards the other aircraft while not sensing it.

Using Proposition 1, we will now show that two aircraft are unable to collide if they use the proposed control law.

Proposition 2: Two aircraft with the dynamics given by Equation 1 and the control laws given by Equations 11, 13 and 15 will not collide.

Proof: The proof is done in a similar way as proposed in [12], but care has to be taken because of the vertical component of the aircraft's velocity. Consider two aircraft $i$ and $j$ about to collide at point $P$ at time $t_{c}$. We will prove that with the proposed control law there is no trajectory that leads to point $P$, that is, both the horizontal and vertical velocities will drive the aircraft away from point $P$ before it is reached.
First, notice that at least one of the two aircraft has to move towards the collision point. Let's assume, without loss of generality, it is aircraft $i$. Two cases have to be studied: first, the case where the camera is pointing towards the collision point $P$. As a result $\beta_{a_{j}} \beta_{d_{j}}=1$. A second case is when $0<\beta_{a_{j}} \beta_{d_{j}}<1$.

For the first case, when $\beta_{a_{j}} \beta_{d_{j}}=1$, the horizontal velocity is directed towards the collision point. We will prove that $u_{i}$ will change the heading of the aircraft early enough so that the aircraft moves away from point $P$ at time $t_{c}$. Consider the situation a short moment before the two aircraft collide and are separated by a small distance $2 R$. Because the maximum speed of the aircraft is $v_{\max }$, the minimum time $\Delta t$ before the two aircraft collide is given by:

$$
\Delta t=\frac{R}{v_{\max }} .
$$

Because of $\beta_{a_{j}} \beta_{d_{j}}=1$, the turning rate is bounded above by the interaction between aircraft $i$ and $j$ :

$$
u_{i}=\sum_{j} \beta_{a_{j}} \beta_{d_{j}} \frac{-\pi v_{\max }}{d_{i j}}<\frac{-\pi v_{\max }}{d_{i j}}<0
$$

The change in heading between time $t \in\left[t_{c}-\Delta t, t_{c}\right]$ is as a result also bounded:

$$
\begin{aligned}
\left|\Delta \theta_{i}\right| & =\left|\int_{t_{c}-\Delta t}^{t_{c}} u_{i} d t\right| \\
& \geq\left|\int_{t_{c}-\Delta t}^{t_{c}} \frac{-2 \pi v_{\max }}{d_{i j}} d t\right| \\
& \geq\left|\int_{t_{c}-\Delta t}^{t_{c}} \frac{-2 \pi v_{\max }}{2 R} d t\right| \\
& =\left|\frac{-\pi v_{\max }}{R} \Delta t\right|=\pi
\end{aligned}
$$


As a result aircraft $i$ will do more than half a turn, thus moving away from the collision point. Thus they could only collide if the two aircraft come closer on the vertical axis. But because $\beta_{a_{j}} \beta_{d_{j}}=1$, the vertical velocity for aircraft $i$ can only make it move away from the collision point as only the component $\nabla V_{i j}^{a v o i d}$ remains in $\nabla V_{i j}^{t o t}$. This can be verified by computing the sign of the scalar product between the vertical velocity $l_{i} \mathbf{z}$ and the vector between the two aircraft $\mathbf{q}_{i}-\mathbf{q}_{j}$. Because $l_{i}$ is monotonic and odd as function of $-\nabla V_{i}^{t o t} \mathbf{z}$, it is possible to ignore the saturation as it does not influence the sign of the scalar product. The following result is obtained:

$$
\begin{aligned}
& \operatorname{sign}\left(l_{i} \mathbf{z} \cdot\left(\mathbf{q}_{i}-\mathbf{q}_{j}\right)\right) \\
& =\operatorname{sign}\left(\left(-K_{z} \nabla V_{i}^{\text {tot }} \cdot \mathbf{z}\right)\left(\mathbf{z} \cdot\left(\mathbf{q}_{i}-\mathbf{q}_{j}\right)\right)\right) \\
& =\operatorname{sign}\left(\left(-K_{z} \nabla V_{i}^{\text {tot }} \cdot \mathbf{z}\right)\left(z_{i}-z_{j}\right)\right) \\
& =\operatorname{sign}\left(\left(-K_{z} \nabla V_{i}^{\text {avoid }} \cdot \mathbf{z}\right)\left(z_{i}-z_{j}\right)\right) \\
& =\operatorname{sign}\left(\frac{K_{z} K_{\text {avoid }}}{\left(\left\|\mathbf{q}_{i}-\mathbf{q}_{j}\right\|-2 r\right)^{2}} \frac{\left(z_{i}-z_{j}\right)}{\left\|\mathbf{q}_{i}-\mathbf{q}_{j}\right\|}\left(z_{i}-z_{j}\right)\right) \geq 0 .
\end{aligned}
$$

The vertical speed vector is, if not zero, in the same direction as the vector that goes from aircraft $j$ (encounter) to aircraft $i$. This shows that the vertical velocity will not drives aircraft $i$ towards aircraft $j$.

The second case is when $0<\beta_{a_{j}} \beta_{d_{j}}<1$. This is only due to $\beta_{a_{j}}<1$ because the distance is considered small, thus $\beta_{d_{j}}=1$. In this case, the horizontal velocity is already moving the aircraft away from the collision point. As for the vertical velocity $l_{i}$, one has to consider an $R$ small enough so that:

$$
\left|\beta_{a_{j}} \beta_{d_{j}} \nabla V_{i j}^{\text {avoid }} \mathbf{z}\right| \gg\left|\left(1-\max _{j}\left(\beta_{a_{j}} \beta_{d_{j}}\right)\right) \nabla V_{i}^{\text {nav }} \mathbf{z}\right|
$$

The reasoning is then the same as for Equation 24. As a result, the vertical velocity component $l_{i}$ will try to drive the aircraft away from the collision point. There exists such small $R$ that Equation 25 is satisfied, with two corner cases that require further attention. One corner case is if the two aircraft are at the same height, in which case $\nabla V_{i j}^{a v o i d} \cdot \mathbf{z} \approx 0$. Define $\phi$ the angle between the vector $\nabla V_{i j}^{a v o i d}$ and the vector $z$ so that:

$$
\begin{array}{rlcl}
-\nabla V_{i j}^{\text {avoid }} \cdot \mathbf{z} & = & \left\|-\nabla V_{i j}^{\text {avoid }}\right\|\|\mathbf{z}\| \cos \phi \\
& = & \| \frac{K_{\text {avoid }} K_{z}}{\left(\frac{\left.q_{i}-q_{j} \|-2 r\right)^{2}}{\|} \frac{q_{i}-q_{j}}{\left\|q_{i}-q_{j}\right\|} \| \cos \phi\right.} \\
& = & \frac{K_{\text {avoid }} K_{z}}{\left(\left\|q_{i}-q_{j}\right\|-2 r\right)^{2}}\left\|\frac{q_{i}-q_{j}}{\left\|q_{i}-q_{j}\right\|}\right\| \sin \left(\frac{\pi}{2}-\phi\right) \\
& = & K_{\text {avoid }} K_{z} \frac{\sin \left(\frac{\pi}{2}-\phi\right)}{\left(\left\|q_{i}-q_{j}\right\|-2 r\right)^{2}} \\
& = & K_{\text {avoid }} K_{z} \frac{\sin \left(\frac{\pi}{2}-\phi\right)}{R^{2}}
\end{array}
$$

By choosing $R=\frac{\pi}{2}-\phi$ and taking he limit $\phi \rightarrow \pi / 2$ we obtain:

$$
\lim _{\phi \rightarrow \pi / 2}\left|K_{\text {avoid }} K_{z} \frac{\sin \left(\frac{\pi}{2}-\phi\right)}{\left(\frac{\pi}{2}-\phi\right)^{2}}\right|=\infty
$$

thus the avoidance component $\nabla V_{i j}^{\text {avoid }}$ grows faster than it decreases, being almost perpendicular to the vertical axis. And
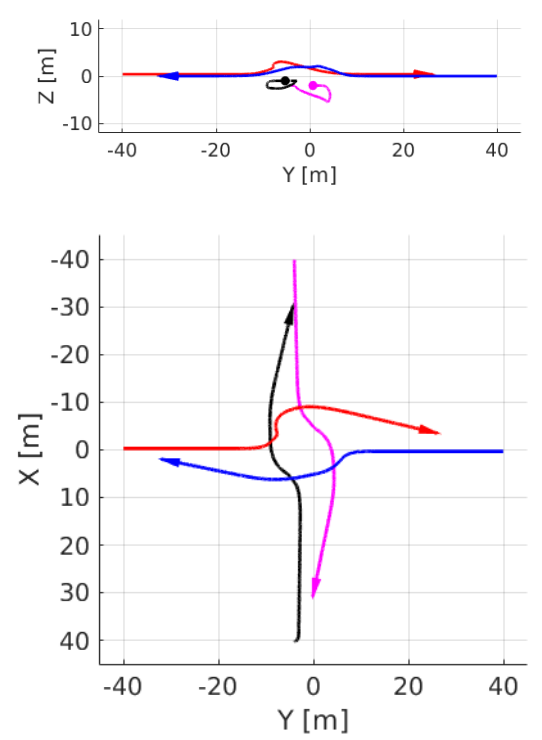

Fig. 4. Example of trajectories obtained with 4 aircraft.

because of Equation 24 we know that the vertical component will always drive aircraft $i$ away. If $\nabla V_{i j}^{\text {avoid }} \cdot \mathbf{z}=0$, the case degenerates to the 2D case treated in [12] and the collision avoidance is also guaranteed. Another corner case is if $\beta_{a_{j}}=$ 0 , because $\beta_{a_{j}} \beta_{d_{j}} \nabla V_{i j}^{a v o i d}=0$. But this case is treated in Proposition 1 with the margin angle $\rho>0$.

As a result, aircraft $i$ cannot reach the collision point. There are two possibilities for aircraft $j$, it does either not see aircraft $i$ and is moving away from $i$ as proven in Proposition 1, or it does see it and the same reasoning can be done as is done for aircraft $i$. As a result there is a contradiction between the existence of a collision point $P$ and the incapacity of both aircraft to reach that point. This leads to the conclusion that such a collision point cannot exist and that the two aircraft cannot collide with each other.

\section{Simulation}

In this section we will present simulations to show the effectiveness of the presented algorithm in comparison to the algorithm presented in [12]. The turning rate command will be studied in depth as it is the only input that is not bounded in both algorithms. Although both algorithms rely on the fact that it can be infinite to prove collision avoidance, it usually remains bounded and depends on the parameters used. Keeping it low is thus of great importance.

We simulated the encounter of two aircraft in three different scenarios and the obtained trajectories are shown in Figure 3. The parameters used are: $v_{+}=0.5, v_{-}=0.4, \alpha_{s}=110^{\circ}$, $r=1, R_{a}=4, R_{S}=20, K_{a}=1.0, K_{z}=1.0, K_{\text {nav }}=0.2$, $K_{\text {avoid }}=80, \rho=5^{\circ}$. The simulations were performed with a time step of 0.05 seconds.

Both algorithms show different behaviors: generally speaking, taking advantage of the third dimension enhances the trajectories by making them smoother in most cases (compare Figures $3 a$ and $3 c$ with Figures $3 d$ and $3 f$ ). Since for the 


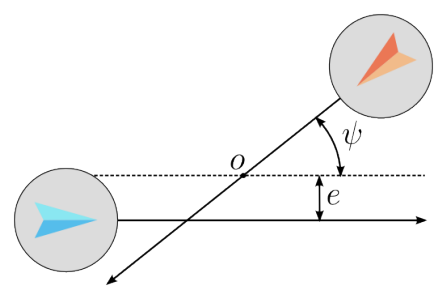

Fig. 5. Comparison of the average turning rate for the $2 \mathrm{D}$ and $3 \mathrm{D}$ implementations for our case scenario.
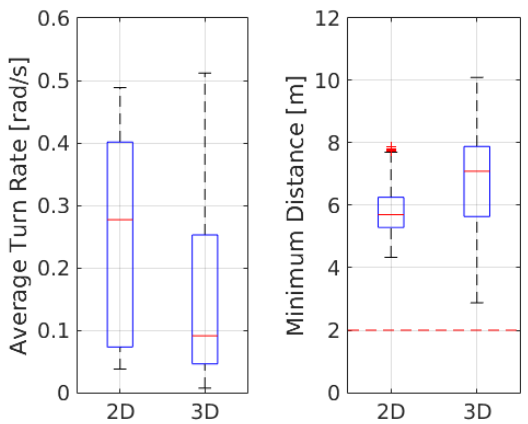

Fig. 6. Left boxplot: Comparison of the average turning rate command input for the 2D and 3D implementations. Right boxplot: Minimum distances between two aircraft during the simulations. The red dashed line is the distance for which the two aircraft are considered colliding.

3D collision avoidance algorithm the potential field has been modified to add $V_{i j}^{a v o i d}$, the turning rate command will tend to react strongly around the obstacle. As a result, the 3D collision avoidance algorithm does not always produce the most smooth trajectories, as for example shown in Figure 3b. Our algorithm is also capable of avoiding several aircraft. An example is shown in Figure 4.

Another scenario was designed to show that the presented algorithm reduces further the average turning rate when compared to the algorithm reported in [12]. The scenario is based on three parameters: the distance between tracks $e$, the angle between tracks $\psi$ (both shown on Figure 5) and the height between tracks $h$. The aircraft aim to stay at their initial height and to follow their track. The parameters are chosen randomly with uniform distribution $\mathcal{U}$ as follows: $e \sim 12(\mathcal{U}-0.5), \phi \sim 1.6 \pi(\mathcal{U}-0.5)$ and $h \sim 8 r(\mathcal{U}-0.5)$. The simulations were performed 1000 times. For each run, the root mean squared turning rate command over the whole simulation run was computed. The result are presented as boxplots in Figure 6. The novel 3D algorithm does perform better than the 2D version from [12] in terms of the average turning rate with 3 times smaller median. This shows that the algorithm trades vertical movement for smoother horizontal curves. However, the novel algorithm does sometimes have a larger average turning rate, due to the addition of the avoidance potential field resulting in more aggressive maneuvers. The minimum distance between aircraft during the whole simulation was also recorded and for none of the simulations the minimum distance went below the collision distance of $2 r$ (see Figure 6).

\section{CONCLUSION}

We presented a provably safe collision avoidance algorithm based on potential fields for fixed-wing UAVs with constrained FOV sensors such as cameras. We demonstrated the effectiveness of our method with several simulations, including one with randomized trajectories covering a large set of possible configurations. This work extends the work presented in [12] and show that the turning rate command is reduced on average when leveraging the third dimension.

Possible future work includes the use of encountered aircraft's velocity to enhance the avoidance behavior. One possibility is to modify another class of algorithms based on the concept of Velocity Obstacle by restricting the motion according to sensing constraints. Another future direction involves bounding the turning rate command input while keeping the guarantees of collision avoidance.

\section{REFERENCES}

[1] S. Weiss, M. W. Achtelik, S. Lynen, M. Chli, and R. Siegwart, "Realtime Onboard Visual-Inertial State Estimation and Self-Calibration of MAVs in Unknown Environments," in IEEE International Conference on Robotics and Automation, 2012, pp. 957-964.

[2] M. Achtelik, M. Achtelik, S. Weiss, and R. Y. Siegwart, "Onboard IMU and Monocular Vision Based Control for MAVs in Unknown In- and Outdoor Environments," in IEEE International Conference on Robotics and Automation, 2011, pp. 3056-3063.

[3] C. Forster, M. Faessler, F. Fontana, M. Werlberger, and D. Scaramuzza, "Continuous on-board monocular-vision-based elevation mapping applied to autonomous landing of micro aerial vehicles," in IEEE International Conference on Robotics and Automation, 2015, pp. 111118.

[4] J. Zufferey, A. Beyeler, and D. Floreano, "Autonomous flight at low altitude with vision-based collision avoidance and GPS-based path following," in IEEE International Conference on Robotics and Automation, 2010, pp. 3329-3334.

[5] Y. Shaowu, S. S. A., S. Konstantin, and Z. Andreas, "Autonomous Landing of MAVs on an Arbitrarily Textured Landing Site Using Onboard Monocular Vision," Journal of Intelligent \& Robotic Systems, pp. 27-43, 2013.

[6] B. Fankhauser, L. Makarem, and D. Gillet, "Collision-free intersection crossing of mobile robots using decentralized navigation functions on predefined paths," in IEEE International Conference on Cybernetics and Intelligent Systems, Sept 2011, pp. 392-397.

[7] C. Carbone, U. Ciniglio, F. Corraro, and S. Luongo, "A novel 3D geometric algorithm for aircraft autonomous collision avoidance," in IEEE Conference on Decision and Control, 2006, pp. 1580-1585.

[8] D. E. Chang and J. E. Marsden, "Gyroscopic forces and collision avoidance with convex obstacles," in New trends in nonlinear dynamics and control and their applications, 2003, pp. 145-159.

[9] P. Panyakeow and M. Mesbahi, "Decentralized deconfliction algorithms for unicycle UAVs," in American Control Conference, June 2010, pp. 794-799.

[10] P. Conroy, D. Bareiss, M. Beall, and J. van den Berg, "3-D reciprocal collision avoidance on physical quadrotor helicopters with on-board sensing for relative positioning," arXiv preprint arXiv:1411.3794, 2014.

[11] A. Mcfadyen, L. Mejias, P. Corke, and C. Pradalier, "Aircraft collision avoidance using spherical visual predictive control and single point features," in IEEE/RSJ International Conference on Intelligent Robots and Systems, 2013, pp. 50-56.

[12] S. Roelofsen, A. Martinoli, and D. Gillet, "Distributed deconfliction algorithm for unmanned aerial vehicles with limited range and field of view sensors," in American Control Conference, 2015, pp. 4356-4361.

[13] S. Roelofsen, D. Gillet, and A. Martinoli, "Reciprocal collision avoidance for quadrotors using on-board visual detection," in IEEE/RSJ International Conference on Intelligent Robots and Systems, 2015, pp. 4810-4817.

[14] L. Makarem and D. Gillet, "Decentralized coordination of autonomous vehicles at intersections," in IFAC World Congress, vol. 18, no. 1, 2011, pp. $13046-13051$. 